\title{
Interplay between Intrinsic Conformational Propensities and Intermolecular Interactions in Self-Assembly of Short Surfactant-Like Peptides Composed of Leucine/Isoleucine
}

Peng Zhou, ${ }^{\dagger}$ Li Deng, ${ }^{\dagger}$ Yanting Wang, ${ }^{*}{ }^{\dagger}$ Jian R. Lu ${ }^{*,}$ and Hai $\mathrm{Xu}^{*}{ }^{* \dagger}$

${ }^{\dagger}$ State Key Laboratory of Heavy Oil Processing and Centre for Bioengineering and Biotechnology, China University of Petroleum (East China), 66 Changjiang West Road, Qingdao, China. Fax: +86-532-86981569; Tel: +86-532-86981569;

Email: xuh@upc.edu.cn

${ }^{\ddagger}$ Institute of Theoretical Physics, Chinese Academy of Science, 55 East Zhongguancun Road, Beijing, China. Tel:

+86-10-62648749; E-mail: wangyt@itp.ac.cn

${ }^{\S}$ Biological Physics Laboratory, School of Physics and Astronomy, The University of Manchester Institution,

Manchester, M13,9PL, United Kingdom. Tel: +44-161-3063926; E-mail: j.lu.@manchester.ac.uk 


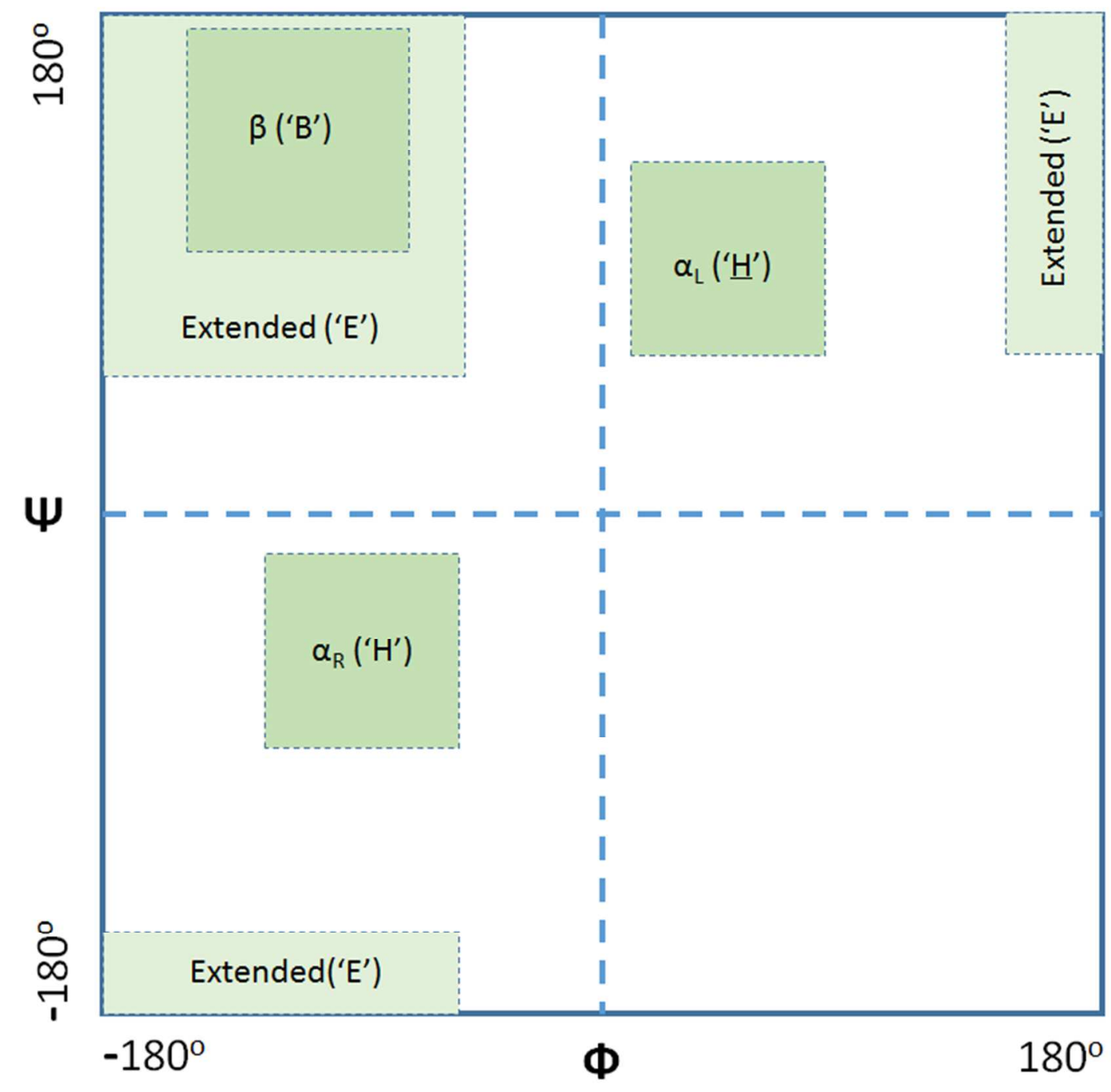

Figure S1. Definition of residue conformations in the Ramachandran map based on ( $\Phi$, $\Psi)$ dihedral angle distributions. The defined regions were $\alpha_{\mathrm{R}}\left(\mathrm{H}^{\prime}\right):-122^{\circ} \leq \Phi \leq-52^{\circ}$ and $-84^{\circ} \leq \Psi \leq-14^{\circ}, \alpha_{\mathrm{L}}\left(\underline{\mathrm{H}}^{\prime}\right): 14^{\circ} \leq \Phi \leq 84^{\circ}$ and $52^{\circ} \leq \Psi \leq 122^{\circ}, \beta\left({ }^{\prime} \mathrm{B}^{\prime}\right):-150^{\circ} \leq \Phi \leq-70^{\circ}$ and $95^{\circ} \leq \Psi \leq 175^{\circ}$, Extended (PPII and near- $\left.\beta,{ }^{\prime} \mathrm{E}\right)$ ): $-180^{\circ} \leq \Phi \leq-50^{\circ}$ and $50^{\circ} \leq \Psi \leq 180^{\circ}$, $-180^{\circ} \leq \Phi \leq-50^{\circ}$ and $-180^{\circ} \leq \Psi \leq-150^{\circ}, 0^{\circ} \leq \Phi \leq 30^{\circ}$ and $50^{\circ} \leq \Psi \leq 180^{\circ}$, and the $\beta$ region was excluded from the Extended region 


\section{GGIGG}

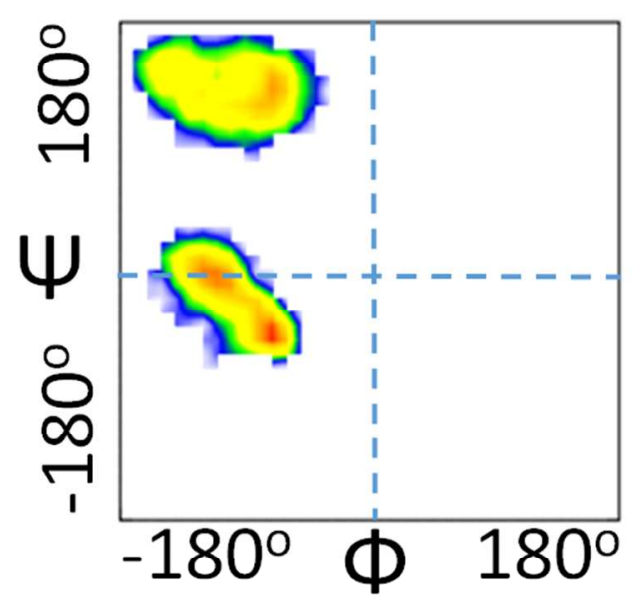

GGLGG

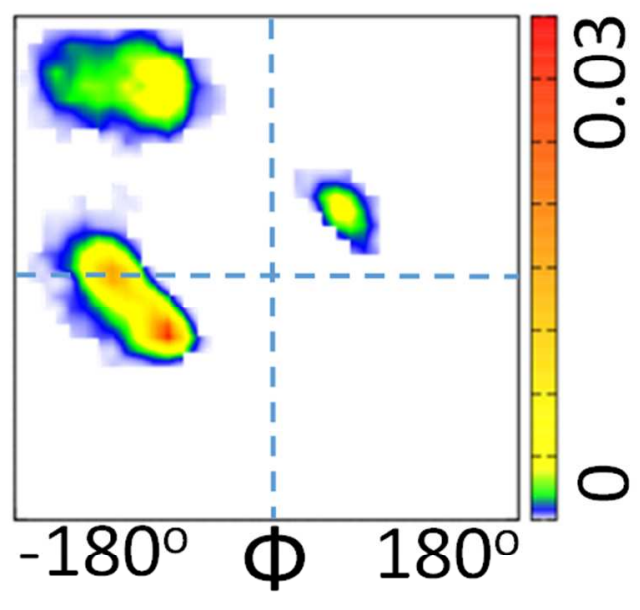

Figure S2. Ramachandran maps of the $\mathrm{X}$ residues in simulations of GGXGG $(\mathrm{X}=\mathrm{I}$ or $\mathrm{L})$ monomers. 


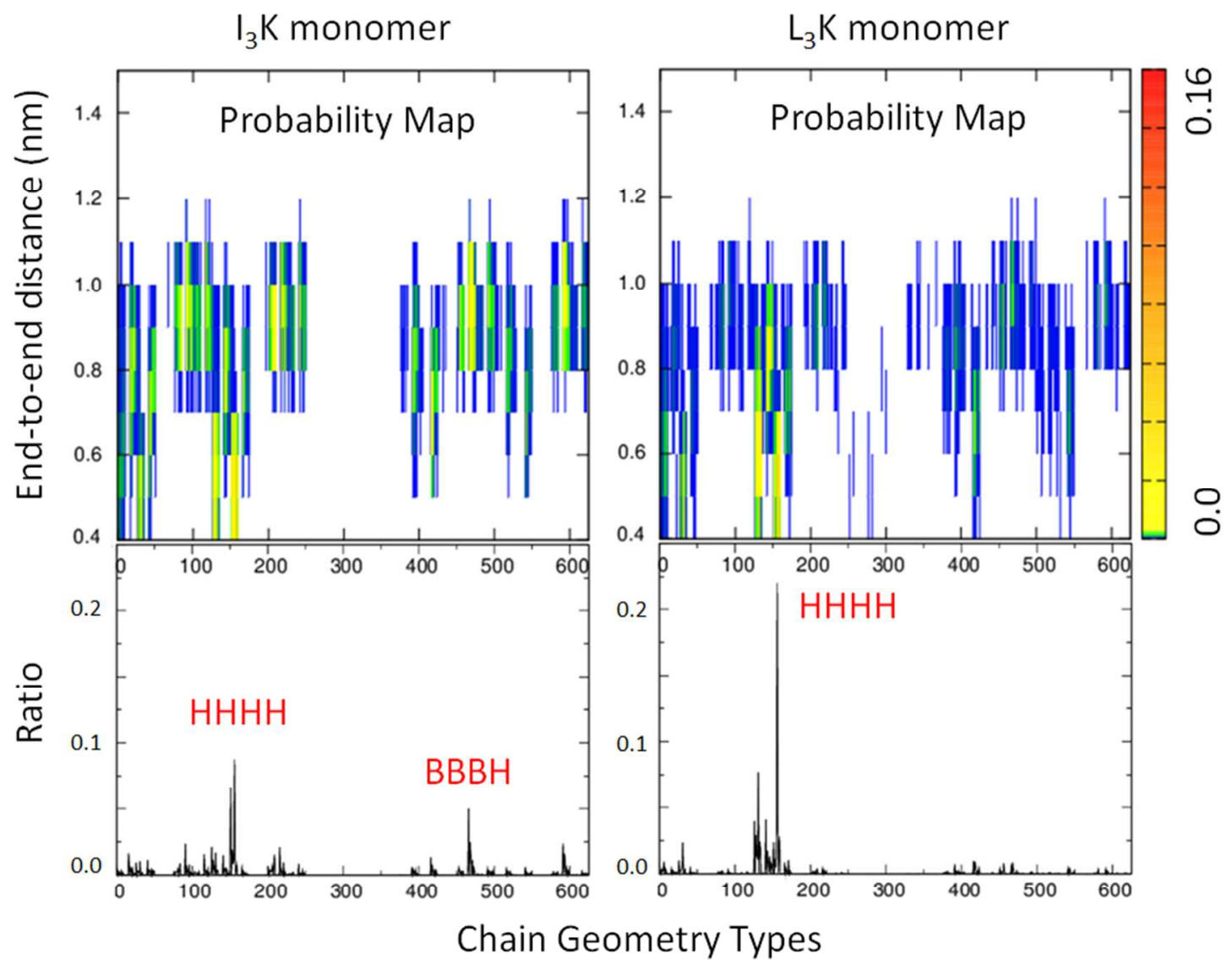

Figure S3. (Top) Probability maps of the end-to-end distance and chain geometry type of $\mathrm{I}_{3} \mathrm{~K}$ and $\mathrm{L}_{3} \mathrm{~K}$ monomers. (Bottom) Chain geometry distributions of $\mathrm{I}_{3} \mathrm{~K}$ and $\mathrm{L}_{3} \mathrm{~K}$ monomers. 


\section{Chain Geometry End-to-end distance}
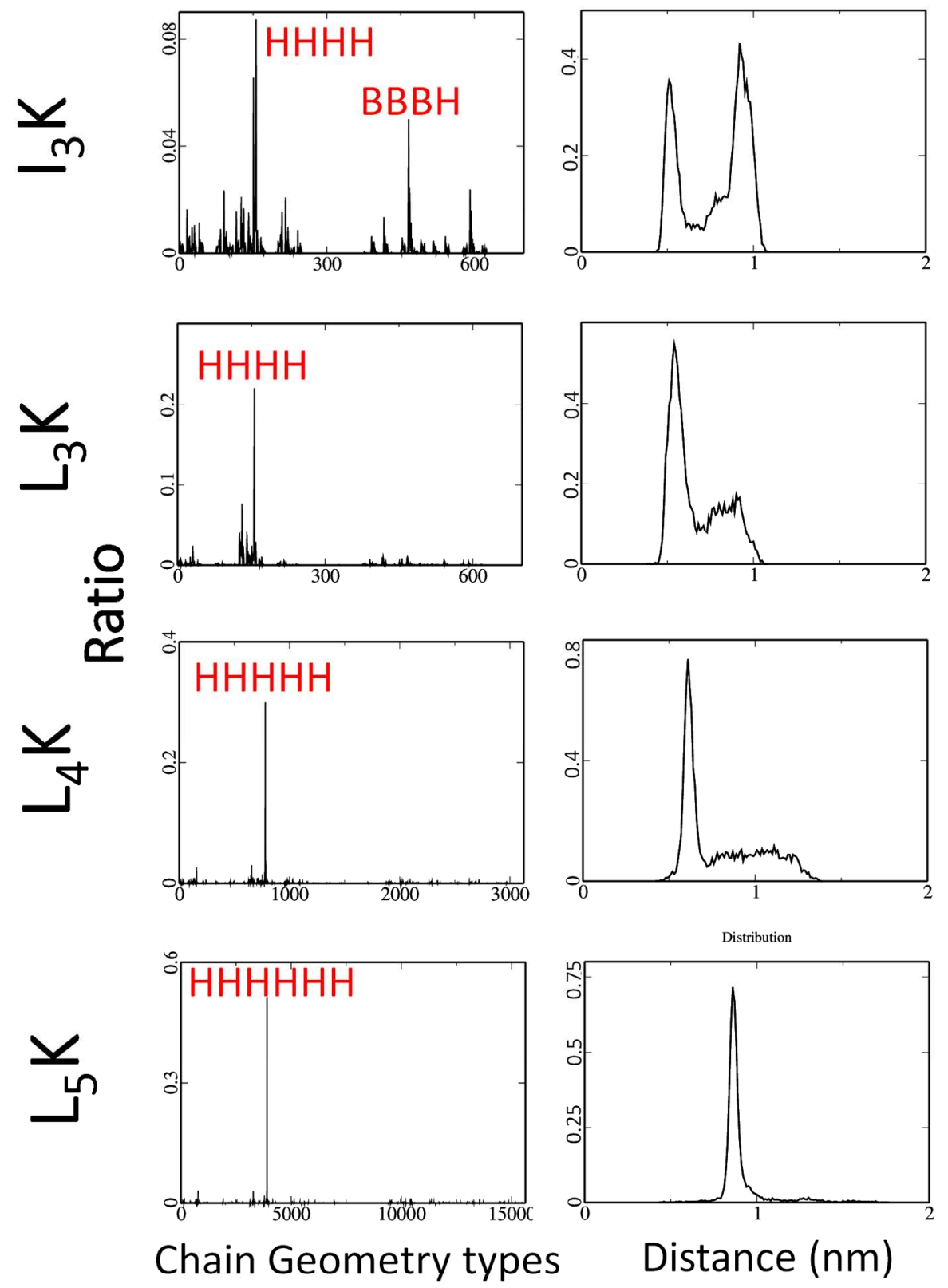

\section{Chain Geometry types Distance $(\mathrm{nm})$}

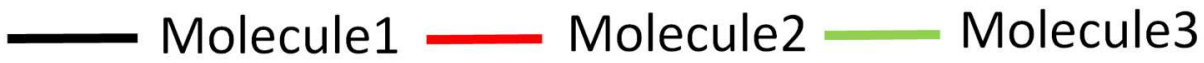

Figure S4. Chain geometry distributions and end-to-end distances of $\mathrm{I}_{3} \mathrm{~K}, \mathrm{~L}_{3} \mathrm{~K}, \mathrm{~L}_{4} \mathrm{~K}$ and $\mathrm{L}_{5} \mathrm{~K}$ monomers. 


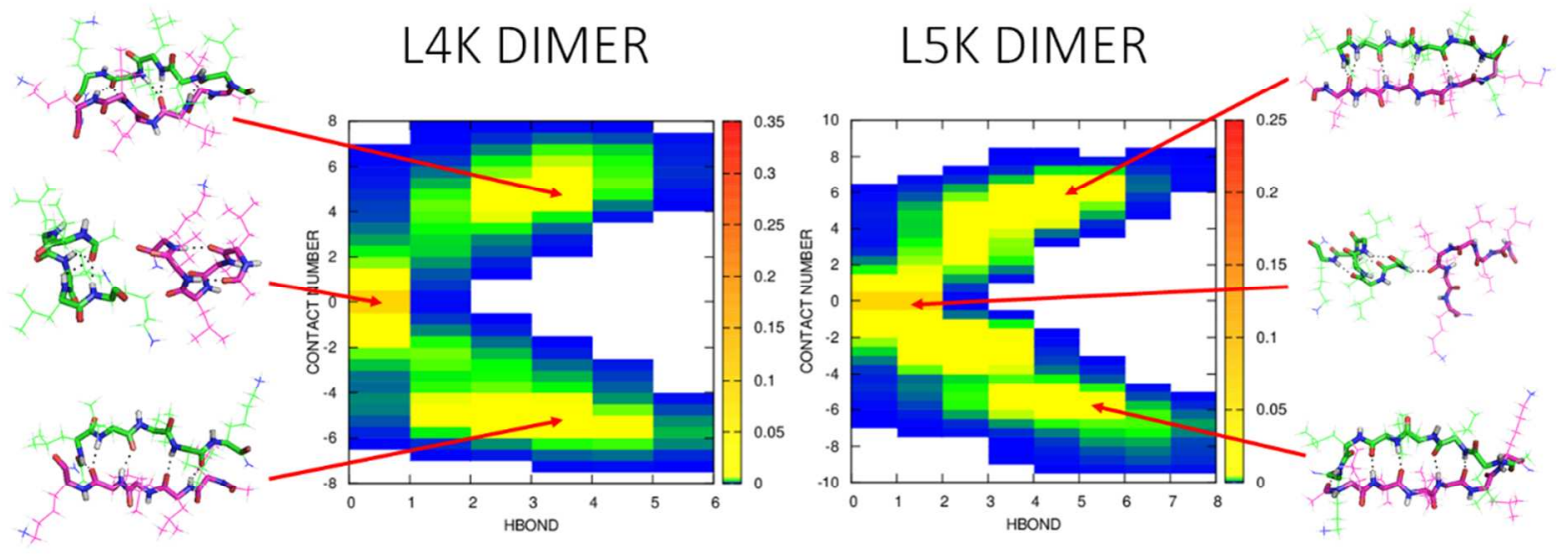

Figure S5. Probability maps of the contact degree and the intermolecular H-bond number of $\mathrm{L}_{4} \mathrm{~K}$ and $\mathrm{L}_{5} \mathrm{~K}$ in dimer simulations and representative intermolecular alignments. 

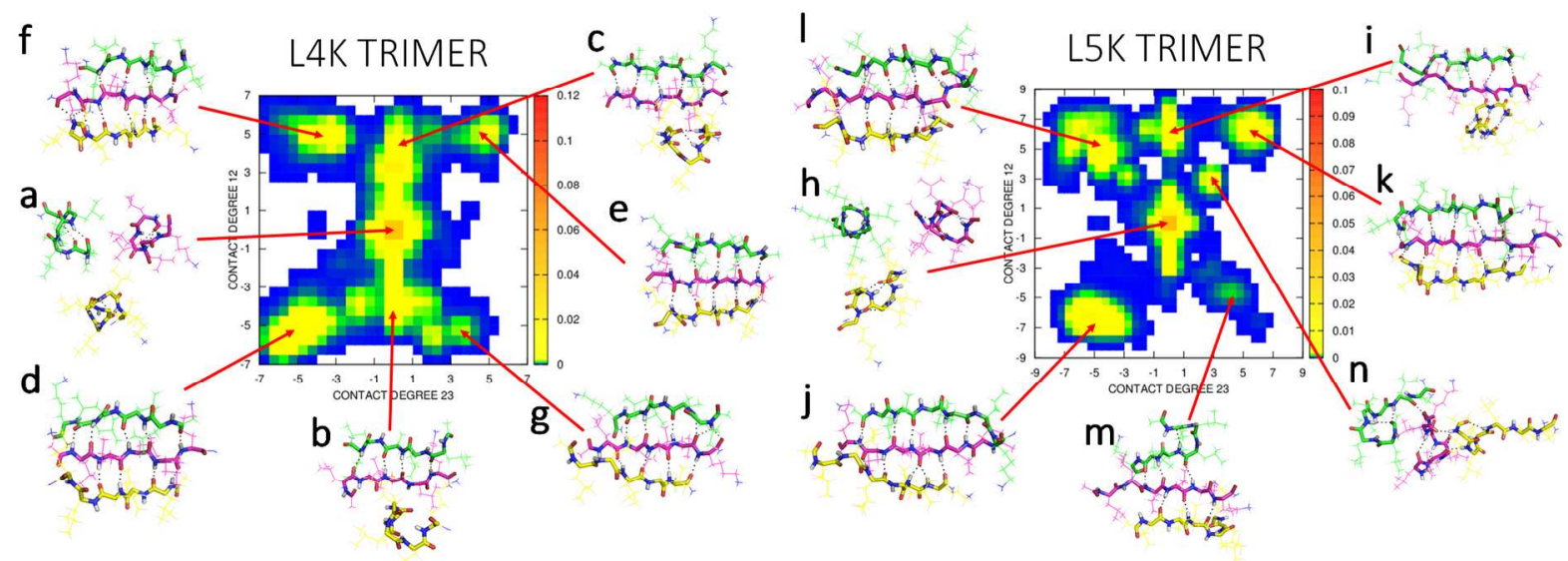

Figure S6. Probability maps of the contact degree of $\mathrm{L}_{4} \mathrm{~K}$ and $\mathrm{L}_{5} \mathrm{~K}$ in trimer simulations.

$\mathrm{L}_{4} \mathrm{~K}$ is found to form trimer aggregates with no intermolecular H-bonding (Structure "a"), dimers with both anti-parallel and parallel H-bonding (Structures "b" and "c"), anti-parallel and parallel trimers (Structures "d" and "e"), and mixed trimers of parallel and anti-parallel alignments (Structures "f" and "g"). $\mathrm{L}_{5} \mathrm{~K}$ also shows six regions, including trimer aggregates without intermolecular H-bonding (Structure "h"), parallel dimers (Structure "i"), anti-parallel and parallel trimers (Structures "j" and "k"), and parallel/anti-parallel mixed trimers (Structures "l" and "m"). There are very few anti-parallel dimers but a large population of anti-parallel trimers (Structure "j"), which implies that the transition from anti-parallel dimers to anti-parallel trimers is quick and complete. Since $\mathrm{L}_{5} \mathrm{~K}$ has a long backbone which also favors the formation of intramolecular H-bonds, some coiled aggregates with both intramolecular H-bonding and intermolecular H-bonding (Structure "n") are observed. 
Table S1. Top 10 chain geometry types of $\mathrm{I}_{3} \mathrm{~K}$ and $\mathrm{L}_{3} \mathrm{~K}$ monomers, dimers and trimers simulated at the temperature of $293 \mathrm{~K}$.

\begin{tabular}{|c|c|c|c|c|c|c|}
\hline & \multicolumn{2}{|c|}{ Monomer } & \multicolumn{2}{|c|}{ Dimer } & \multicolumn{2}{|c|}{ Trimer } \\
\hline \multirow{10}{*}{ I3K } & $\mathrm{HHHH}$ & $8.73 \%$ & BBBH & $9.10 \%$ & BBBB & $16.11 \%$ \\
\hline & $\mathrm{HH}-\mathrm{H}$ & $6.57 \%$ & BBBB & $7.68 \%$ & $\mathrm{BBBH}$ & $11.41 \%$ \\
\hline & $\mathrm{BBBH}$ & $5.00 \%$ & $\mathrm{HHHH}$ & $5.87 \%$ & BBBE & $7.28 \%$ \\
\hline & BBBB & $2.47 \%$ & BBBE & $4.02 \%$ & $\mathrm{HHHH}$ & $4.00 \%$ \\
\hline & EBBH & $2.40 \%$ & BBEH & $2.82 \%$ & EBBB & $3.97 \%$ \\
\hline & $-\mathrm{BBH}$ & $2.36 \%$ & $\mathrm{HH}-\mathrm{H}$ & $2.77 \%$ & BBEB & $3.02 \%$ \\
\hline & $\mathrm{H}--\mathrm{H}$ & $2.12 \%$ & $\mathrm{EBBH}$ & $2.56 \%$ & $\mathrm{EBBH}$ & $2.78 \%$ \\
\hline & $\mathrm{HBBH}$ & $2.10 \%$ & EBBB & $2.54 \%$ & $-B B B$ & $2.38 \%$ \\
\hline & HH-B & $1.91 \%$ & BBEB & $2.05 \%$ & BBEH & $2.38 \%$ \\
\hline & $\mathrm{H}-\mathrm{HH}$ & $1.69 \%$ & HBBB & $1.74 \%$ & EBBE & $1.87 \%$ \\
\hline \multirow{10}{*}{ L3K } & $\mathrm{HHHH}$ & $22.03 \%$ & $\mathrm{HHHH}$ & $22.11 \%$ & $\mathrm{HHHH}$ & $20.46 \%$ \\
\hline & $\mathrm{H}-\mathrm{HH}$ & $7.69 \%$ & $\mathrm{H}-\mathrm{HH}$ & $7.59 \%$ & $\mathrm{H}-\mathrm{HH}$ & $6.49 \%$ \\
\hline & $\mathrm{H}-\mathrm{BH}$ & $4.09 \%$ & $\mathrm{H}-\mathrm{BH}$ & $4.05 \%$ & $\mathrm{H}-\mathrm{BH}$ & $3.52 \%$ \\
\hline & $\mathrm{H}-\mathrm{-H}$ & $3.99 \%$ & $\mathrm{H}-\mathrm{-H}$ & $2.98 \%$ & $\mathrm{H}--\mathrm{H}$ & $2.56 \%$ \\
\hline & H--B & $2.90 \%$ & $\mathrm{H}-\mathrm{BB}$ & $2.62 \%$ & $\mathrm{HHH}-$ & $2.19 \%$ \\
\hline & HHHE & $2.84 \%$ & $\mathrm{HH}-\mathrm{H}$ & $2.40 \%$ & $\mathrm{H}-\mathrm{EH}$ & $2.08 \%$ \\
\hline & $\mathrm{HHH}-$ & $2.79 \%$ & $-\mathrm{HHH}$ & $2.33 \%$ & $-\mathrm{HHH}$ & $2.03 \%$ \\
\hline & $\mathrm{H}-\mathrm{HB}$ & $2.42 \%$ & $\mathrm{HHH}-$ & $2.25 \%$ & $\mathrm{H}-\mathrm{BB}$ & $2.00 \%$ \\
\hline & $\mathrm{HH}-\mathrm{H}$ & $2.37 \%$ & H--B & $1.90 \%$ & BBBB & $1.92 \%$ \\
\hline & $-\mathrm{HHH}$ & $2.36 \%$ & $\mathrm{H}-\mathrm{HB}$ & $1.62 \%$ & HBBB & $1.88 \%$ \\
\hline \multirow{10}{*}{ L4K } & $\mathrm{HHHHH}$ & $30.66 \%$ & $\mathrm{HHHHH}$ & $22.94 \%$ & $\mathrm{HHHHH}$ & $17.68 \%$ \\
\hline & $\mathrm{HHHH}-$ & $3.37 \%$ & $\mathrm{HHHH}-$ & $3.16 \%$ & BBBBB & $6.95 \%$ \\
\hline & $\mathrm{H}-\mathrm{HHH}$ & $2.91 \%$ & $\mathrm{HHHH}$ & $2.81 \%$ & BBBBH & $6.00 \%$ \\
\hline & $-\mathrm{HHHH}$ & $2.80 \%$ & HHHHE & $2.26 \%$ & BBBBE & $2.62 \%$ \\
\hline & HHHHE & $2.78 \%$ & $\mathrm{H}-\mathrm{HHH}$ & $2.12 \%$ & HBBBB & $2.22 \%$ \\
\hline & $\mathrm{HHH}-\mathrm{H}$ & $1.99 \%$ & $\mathrm{H}-\mathrm{H}-\mathrm{H}$ & $1.80 \%$ & $\mathrm{HHHH}-$ & $2.16 \%$ \\
\hline & $\mathrm{H}-\mathrm{H}-\mathrm{H}$ & $1.40 \%$ & $\mathrm{HHH}-\mathrm{H}$ & $1.70 \%$ & EBBBB & $2.02 \%$ \\
\hline & $\mathrm{HH}-\mathrm{HH}$ & $1.21 \%$ & $\mathrm{H} \underline{\mathrm{H}} \mathrm{H}-\mathrm{H}$ & $1.28 \%$ & $-\mathrm{HHHH}$ & $1.80 \%$ \\
\hline & $\mathrm{H}--\mathrm{BH}$ & $0.86 \%$ & $\mathrm{BH} \underline{H} \mathrm{HH}$ & $1.09 \%$ & $\mathrm{H}-\mathrm{HHH}$ & $1.48 \%$ \\
\hline & $\mathrm{H}-\mathrm{HBH}$ & $0.82 \%$ & $\mathrm{HH}-\mathrm{HH}$ & $1.08 \%$ & EBBBH & $1.47 \%$ \\
\hline \multirow{3}{*}{ L5K } & $\mathrm{HHHHHH}$ & $45.33 \%$ & $\mathrm{HHHHHH}$ & $26.49 \%$ & $\mathrm{HHHHHH}$ & $17.41 \%$ \\
\hline & НHНHН- & $6.06 \%$ & $\mathrm{H}-\mathrm{HHHH}$ & $3.65 \%$ & BBBBBB & $8.63 \%$ \\
\hline & $\mathrm{HHHHH}$ & $4.29 \%$ & HHHHH- & $2.54 \%$ & BBBBBH & $4.47 \%$ \\
\hline
\end{tabular}




\begin{tabular}{cccccc} 
HHHHHE & $3.95 \%$ & HHHHHE & $1.85 \%$ & BBBBBE & $3.85 \%$ \\
$\mathrm{H}-\mathrm{HHHH}$ & $3.43 \%$ & $\mathrm{HAHHHH}$ & $1.43 \%$ & EBBBBB & $2.00 \%$ \\
$\mathrm{HHHH}-\mathrm{H}$ & $3.29 \%$ & $\mathrm{HHHHH}$ & $1.28 \%$ & $\mathrm{HHHHH}-$ & $1.75 \%$ \\
$\mathrm{HH}-\mathrm{HHH}$ & $1.75 \%$ & $\mathrm{BH} \underline{\mathrm{H} H}-\mathrm{H}$ & $1.28 \%$ & $\mathrm{H}-\mathrm{HHBE}$ & $1.31 \%$ \\
$\mathrm{HHHHHB}$ & $1.26 \%$ & $\mathrm{BH} \underline{\mathrm{H}} \underline{\mathrm{H}} \mathrm{H}$ & $1.17 \%$ & $\mathrm{HHHHHE}$ & $1.15 \%$ \\
$\mathrm{HHH}-\mathrm{HH}$ & $1.25 \%$ & $\mathrm{HHHH}-\mathrm{H}$ & $1.06 \%$ & $\mathrm{BBBHBH}$ & $1.11 \%$ \\
$\mathrm{H}-\mathrm{HHH}-$ & $0.64 \%$ & $\mathrm{BBBBBH}$ & $1.02 \%$ & $\mathrm{EBBBBH}$ & $1.08 \%$ \\
\hline
\end{tabular}

